J. Radtke

J. Linseisen

G. Wolfram

\section{Phenolsäurezufuhr Erwachsener in einem bayerischen Teilkollektiv der Nationalen Verzehrsstudie}

Phenolic acid intake of adults in a Bavarian subgroup of the national food consumption survey

\begin{abstract}
Zusammenfassung Phenolsäuren, im wesentlichen Hydroxyzimtsäuren und Hydroxybenzoesäuren, sind als sekundäre Pflanzenstoffe in Lebensmitteln pflanzlichen Ursprungs weit verbreitet. Aufgrund ihrer antioxidativen und antikanzerogenen Wirkung könnten sie an der koronar- und krebsprotektiven Wirkung einer an Obst und Gemüse reichen Ernährung beteiligt sein. Angaben zum Gehalt von Phenolsäuren in Lebensmitteln sind kaum in Nährwert-Tabellen enthalten, Zufuhrdaten sind nicht bekannt. Deshalb wurden mit Hilfe einer neu erstellten Datenbank zum Phenolsäuregehalt von Lebensmitteln (Literaturwerte) 7-Tage Ernährungsprotokolle von 63 Frauen und 56 Männer eines bayerischen Teilkollektivs (Alter 19-49 Jahre) der Nationalen Verzehrsstudie ausgewertet.

Die mittlere Phenolsäure-Zufuhr des Gesamtkollektivs beträgt $222 \mathrm{mg} / \mathrm{d}$ bei einer relativ großen
\end{abstract}

Dipl. oec. troph. J. Radtke

J. Linseisen - G. Wolfram Institut für Ernährungswissenschaft der TU München

85350 Freising-Weihenstephan
Schwankungsbreite. Unter den betrachteten Phenolsäuren dominiert die Kaffeesäure (206 mg/d), die Zufuhr der anderen Phenolsäuren bewegt sich zwischen 0,2 (Gentisinsäure) und 5,2 mg/d (Ellagsäure). Die Summe der Hydroxybenzoesäuren beträgt $11 \mathrm{mg} / \mathrm{d}$ und die Summe der Hydroxyzimtsäuren $211 \mathrm{mg} / \mathrm{d}$. Für die mittlere Zufuhr mehrerer Phenolsäuren sind signifikante Geschlechtsunterschiede vorhanden. Insbesondere für die mittlere Kaffeesäurezufuhr sind die Werte der Frauen $(229 \mathrm{mg} / \mathrm{d})$, bedingt durch den höheren Kaffeekonsum, größer als die der Männer (179 mg/d). In der Altersgruppe „25-49 Jahre“ wird mehr Kaffee getrunken als in der Altersgruppe „19-24 Jahre“ und somit signifikant mehr Kaffeesäure aufgenommen. Die wichtigsten Zufuhrquellen der Phenolsäuren sind der Kaffee mit $92 \%$ der Kaffeesäurezufuhr und die Lebensmittelgruppe Obst/produkte/-säfte mit $75 \%$ der Salicylsäure- und $59 \%$ der p-Cumarsäure-Zufuhr.

Phenolsäuren werden somit in beträchtlichen Mengen mit der Nahrung zugeführt. Nachdem sowohl in vitro als auch im Tierversuch antioxidative und antikanzerogene Wirkungen von Phenolsäuren nachgewiesen wurden, bleibt es epidemiologischen Studien überlassen, ob sich ein Zusammenhang zwischen phenolsäurereicher Ernährung und vermindertem Risiko für koronare Herzerkrankungen und Krebs beim Menschen bestätigen läßt.

Summary Phenolic acids, essentially hydroxycinnamic acids and hydroxybenzoic acids, are secondary plant products and commonly found in plant derived foodstuff. The antioxidant and anticarcinogenic properties of phenolic acids could be one of the facts to explain the inverse association between fruit and vegetable intake and the incidence of coronary heart disease and cancer, respectively, as found in epidemiologic studies. Phenolic acids are rarely listed in food composition tables and there are no dietary intake data available. Consequently, a data base containing the phenolic acid content of foods (literatur data) was built and 7-d dietary protocols of 63 women and 56 men of a Bavarian subpopulation (age 19-49 years) of the German National Food Consumption Survey (NVS) were evaluated.

The average phenolic acid intake of men and women is $222 \mathrm{mg} / \mathrm{d}$ within a large range. The dominating one within all the phenolic acids is clearly caffeic acid $(206 \mathrm{mg} / \mathrm{d})$; the intake of the other phenolic acids amounts to 0.2 (gentisic acid) up to $5.2 \mathrm{mg} / \mathrm{d}$ (ellagic acid). The sum of hydroxybenzoic acids and hydro- 
xycinnamic acids amounts to $11 \mathrm{mg} / \mathrm{d}$ and $211 \mathrm{mg} / \mathrm{d}$, respectively. Significant sex differences are found for some of the phenolic acids. Especially, the average intake of caffeic acid of women $(229 \mathrm{mg} / \mathrm{d})$ is higher than that of men $(179 \mathrm{mg} / \mathrm{d})$ caused by the high amount of coffee consumption. The age group "25-49 years" is consuming more coffee than the age group "19-24 years" and, therefore, reveals a significantly higher intake of caffeic acid. The major sources of phenolic acids are coffee with $92 \%$ of the caffeic acid intake and fruits (including fruit products and juices) with $75 \%$ of the salycilic acid and $59 \%$ of the p-coumaric acid intake.

Consequently, phenolic acids are consumed in considerable amounts with food. Since antioxidant and anticarcinogenic properties of phenolic acids are already proven in in vitro as well as in animal experiments, epidemiologic studies will show whether a high phenolic acid intake goes ahead with a reduced risk for coronary heart disease or cancer in humans.

Schlüsselwörter Phenolsäuren Hydroxyzimtsäuren - Hydroxybenzoesäuren - Antioxidans Datenbank - Zufuhr

Key words Phenolic acids hydroxycinnamic acids - hydroxybenzoic acids - antioxidant data base - intake

\section{Einleitung}

Eine Ernährung reich an Obst und Gemüse vermindert das Risiko für Herz-Kreislaufkrankheiten und Krebs. Dafür scheinen neben anderen Inhaltsstoffen auch sekundäre Pflanzenstoffe verantwortlich zu sein (2). Einen großen Anteil der sekundären Pflanzenstoffe machen die phenolischen Verbindungen aus. Es handelt sich dabei vor allem um Flavonoide und Phenolsäuren bzw. deren Derivate. Neben den Flavonoiden, die gegenwärtig im Vordergrund des wissenschaftlichen Interesses stehen, besitzen auch die Phenolsäuren, im wesentlichen Hydroxyzimt- und Hydroxybenzoesäuren, antioxidative und antikanzerogene Wirkung (11).

Die Hydroxyzimtsäure-Derivate findet man in pflanzlichen Lebensmitteln ubiquitär verbreitet und in teilweise beträchtlichen Konzentrationen; Hydroxybenzoesäuren liegen häufig nur in geringen Mengen vor. Die „Grundkörper" der Hydroxyzimtsäuren sind neben der Kaffeesäure die p-Cumarsäure, Ferulasäure und Sinapinsäure. In freier Form treten sie in der Pflanze aber gewöhnlich nicht auf, sondern als Ester der Chinasäure oder Weinsäure und mit Glucose verbunden. Das bekannteste Derivat stellt sicherlich die als „Chlorogensäure“ bezeichnete 5-Caffeoylchinasäure dar (4). Die Hydroxybenzoesäuren p-Hydroxybenzoesäure, Protocatechusäure, Vanillinsäure, Salicylsäure und Syringasäure liegen meist in Verbindung mit Glucose vor. Gallussäure findet man dagegen häufig als Ester mit Chinasäure oder Catechinen bzw. als Kondensationsprodukt dieser Verbindungen (4).

Phenolsäuren sind in Nährwert-Tabellen kaum zu finden und es sind auch keine Zufuhr-Daten bekannt. Aus diesem Grund wurde eine Datenbank mit Hydroxyzimtsäure- und Hydroxybenzoesäure-Gehalten pflanzlicher Lebensmittel erstellt und mit deren Hilfe Verzehrsprotokolle der NVS ausgewertet, um so die mittlere tägliche Zufuhr dieser Phenolsäuren abschätzen zu können.

\section{Methodik}

Datenbank

Die Erstellung der Datenbank erfolgte mit Hilfe von Literaturdaten zum quantitativen Gehalt ausgewählter Hydroxyzimtsäuren und Hydroxybenzoesäuren in Lebensmitteln bzw. Getränken pflanzlichen Ursprungs. Die Angaben wurden der bis einschließlich 1995 veröffentlichten Literatur entnommen. Es wurden hierbei nur Werte für den eßbaren Anteil reifer Pflanzen verwendet. Da die Analysenwerte in Abhängigkeit von Sorte, Anbaugebiet und Erntezeitpunkt stark variieren, wurden die Mediane der einzelnen Werte in die Datenbank übernommen. Wenn für das gleiche Lebensmittel mehrere Literaturstellen vorlagen, wurde hierfür der Median errechnet. Ältere Untersuchungen wurden daraufhin überprüft, ob die Werte mit HPLC-Technik ermittelt wurden. War dies nicht der Fall, wurden die Daten nur berücksichtigt, wenn sie von den Werten anderer Autoren nicht stark abwichen oder für ein bestimmtes Lebensmittel den einzigen Wert darstellten.

In die Datenbank wurden die Gehaltsangaben für die „Grundkörper“ der Phenolsäure-Verbindungen wie z.B. Kaffeesäure eingetragen, nachdem vom natürlich vorliegenden Derivat (z.B. Caffeoylchinasäure) die Masse des Säure- bzw. Zuckeranteils abgezogen worden war. Als Ausnahme wird 5-Caffeoylchinasäure zusätzlich aufgeführt. Die Lebensmittel, für welche Analysendaten vorliegen, sind zusammen mit den entsprechenden Phenolsäuren in Tabelle 1 aufgelistet.

\section{Studienkollektiv}

Als Studienkollektiv für die Berechnung der Hydroxyzimtsäure- und Hydroxybenzoesäure-Zufuhr diente ein Teilkollektiv der Nationalen Verzehrsstudie. Es wurden 
Tab. 1 Phenolsäure-Datenbank: aufgenommene Lebensmittel mit Angabe der Gehalte einzelner Phenolsäuren (mg/kg)

\begin{tabular}{|c|c|}
\hline Lebensmittel & Phenolsäure-Gehalt (mg/kg) \\
\hline \multicolumn{2}{|c|}{ Obst und -produkte } \\
\hline Ananas & p-Cum $(15,9)$, Kaff $(3,7)$, Sali $(21,0)$ \\
\hline Ananassaft & p-Cum $(0,4)$, Feru $(1,0)$, Sina $(0,4)$ \\
\hline Apfel & p-Cum $(8,7)$, Feru $(2,2)$, Gent $(2,5)$, Kaff $(57,5)$, Sali $(2,8)$, Syri $(22,5)$ \\
\hline Apfelsaft & p-Cum $(11,7)$, Feru $(0,9)$, Gallu $(1,8), \operatorname{Kaff}(22,6)$, Pcat $(0,5)$ \\
\hline Apfelsine & p-Cum $(5,0)$, Feru $(10,0)$, Kaff $(50,0)$, Sali $(23,0)$ \\
\hline Apfelsinensaft & Feru $(0,5)$, Kaff $(0,5)$, Sina $(0,8)$ \\
\hline Aprikose & p-Cum $(2,7)$, Feru $(4,9)$, Kaff $(85,1)$, Sali $(25,8)$ \\
\hline Aprikosensaft & p-Cum $(0,7)$, p-Hb $(0,3), \operatorname{Kaff}(7,4)$ \\
\hline Birne & p-Cum $(16,6)$, Feru $(0)$, Gent $(5,4)$, Kaff $(76,8)$, Sali $(2,7)$ \\
\hline Birnensaft & p-Cum $(1,3)$, Kaff $(46,6)$ \\
\hline Brombeeren & p-Cum $(6,4)$, Ella $(2010)$, Feru $(4,4)$, Gallu $(19,7)$, p-Hb $(7,9)$, Kaff $(22,8)$, Pcat $(64,9)$, Sali $(18,6)$ \\
\hline Erdbeeren & p-Cum (0), Ella $(226,6)$, Feru $(0,5)$, Gent $(14,5)$, p-Hb $(12,6)$, Kaff $(3,0)$, Pcat $(12,8)$, Sali $(13,6)$, Vani $(1,3)$ \\
\hline Grapefruit & p-Cum $(0)$, Kaff $(40,0)$, Sali $(6,8)$ \\
\hline Heidelbeeren & p-Cum $(1,6)$, Feru $(3,7)$, Gallu $(1,5)$, Kaff $(946,8)$, Pcat $(0)$, Sali $(16,4)$ \\
\hline Himbeeren & p-Cum $(4,8)$, Ella $(650,1)$, Feru $(2,9)$, Gallu $(14,3)$, p-Hb $(20,0)$, Kaff $(3,0)$, Pcat $(31,0)$, Sali $(51,4)$ \\
\hline Himbeersaft & Ella $(9,6)$ \\
\hline Johannisbeeren & p-Cum $(2,9)$, Feru $(1,0)$, Gallu $(0)$, p-Hb $(20,2)$, Kaff $(3,2)$, Pcat $(2,5)$, Sali $(50,6)$ \\
\hline rot & Vani $(4,5)$ \\
\hline $\begin{array}{l}\text { Johannisbeeren } \\
\text { schwarz }\end{array}$ & $\begin{array}{l}\text { p-Cum }(11,5) \text {, Feru }(9,2) \text {, Gallu }(19,8), p-H b(3,0) \text {, Kaff }(39,9) \text {, Pcat }(15,7) \text {, Sali } \\
(30,0)\end{array}$ \\
\hline Johannisb. weiß & Gallu $(20,5), \mathrm{p}-\mathrm{Hb}(12,0)$, Pcat $(3,5)$ \\
\hline Kakifrucht & Feru $(520,0)$, Gallu $(189,9)$, Kaff $(384,0)$, Pcat $(151,6)$ \\
\hline Kirsche sauer & p-Cum $(81,6)$, Feru $(1,6)$, Kaff $(120,6)$, Sali $(8,5)$ \\
\hline Kirsche süß & p-Cum $(149,2)$, Feru $(2,6)$, p-Hb $(4,3)$, Kaff $(160,9)$, Pcat $(0,9)$, Sali $(8,5)$, Vani $(6,5)$ \\
\hline Kiwi & Kaff $(384,0)$, Sali $(1,6)$ \\
\hline Passionsfrucht & Gallu (0), Sali $(1,4)$ \\
\hline Pfirsich & p-Cum $(0,9)$, Feru $(1,2)$, Kaff $(158,5)$, Sali $(5,9)$ \\
\hline Pfirsichsaft & p-Cum $(0,1)$, Feru $(0,5), \mathrm{p}-\mathrm{Hb}(0,1)$, Kaff $(2,0)$ \\
\hline Pflaume & p-Cum $(15,6)$, Feru $(8,9)$, p-Hb $(1,3)$, Kaff $(247,8)$, Sali $(2,1)$, Vani $(7,0)$ \\
\hline Preiselbeeren & Sali $(16,4)$ \\
\hline Rhabarber & p-Cum $(6,0)$, Feru $(0,5)$, Kaff $(0,8)$, Sali $(1,3)$, Sina $(0,9)$ \\
\hline Stachelbeeren & p-Cum $(10,1)$, Feru $(2,3)$, Gallu $(0,5)$, p-Hb $(0,9)$, Kaff $(18,5)$, Pcat $(4,0)$ \\
\hline Traubensaft & p-Cum $(3,9)$, Ella $(14,9)$, Feru $(2,4)$, Gallu $(1,4)$, Kaff $(80,6)$ \\
\hline Weintrauben & $\begin{array}{l}\text { p-Cum }(88,8) \text {, Gallu }(0,2), \text { Gent }(0), \text { p-Hb }(0,04) \text {, Kaff }(350,4) \text {, Pcat }(0,2) \text {, } \\
\text { Sali }(4,7) \text {, Vani }(1,4)\end{array}$ \\
\hline Weintrauben rot & p-Cum $(175,3)$, Gallu $(0,23)$, Gent $(0)$, p-Hb $(0,04)$, Kaff $(700,8)$, Pcat $(0,3)$, Vani $(1,4)$, Sali $(4,7)$ \\
\hline Weintrauben weiß & Gallu $(0,23)$, Gent $(0), \mathrm{p}-\mathrm{Hb}(0,04)$, Sali $(4,7)$, Vani $(1,4)$ \\
\hline Zitrone & p-Cum $(6,0)$, Sali $(1,8)$ \\
\hline
\end{tabular}

\section{Gemüse}

Artischocke

Aubergine

Blumenkohl

Bohnen grün

Broccoli

Brunnenkresse

Champignon

Chinakohl

Chicoree

Endivie

Erbsen

Grünkohl

Gurke

Kartoffel

Kopfsalat

p-Cum $(4,6)$, Feru $(1,0), \operatorname{Kaff}(218,9)$, Sina $(1,2)$

Feru $(8,0)$, Gent $(0)$, Kaff $(329,5)$, Sali $(4,4)$, Vani $(0)$

p-Cum $(18,4)$, Feru $(3,7)$, Gallu $(6,9)$, Kaff $(4,8)$, Pcat $(4,4)$, Sali $(1,6)$, Sina $(22,6)$

Syri $(11,4)$

Sali $(1,1)$

p-Cum $(8,1)$, Feru $(8,3), \operatorname{Kaff}(18,6)$, Sali $(6,5)$, Sina $(6,0)$

$\mathrm{p}-\mathrm{Hb}(1,3)$

Sali $(12,6)$

Feru $(1,7)$, Gallu $(5,2)$, Gent $(8,0)$, Kaff $(4,9)$, Pcat $(3,6)$, Sina $(3,0)$, Syri $(4,6)$

Kaff $(2,2)$, Sali $(10,2)$

Feru $(1,0)$, Kaff $(283,9)$, Sali $(19,0)$

Sali $(0,4)$

p-Cum $(25,0)$, Feru $(200,0)$, Kaff $(210,0)$, Sali (0)

Feru $(15,0)$, Gent $(0)$, Sali $(7,8)$, Vani $(0)$

p-Cum $(1,0)$, Kaff $(28,3)$, Sali $(0)$

Feru $(1,0)$, Kaff $(196,8)$, Sali $(0)$ 


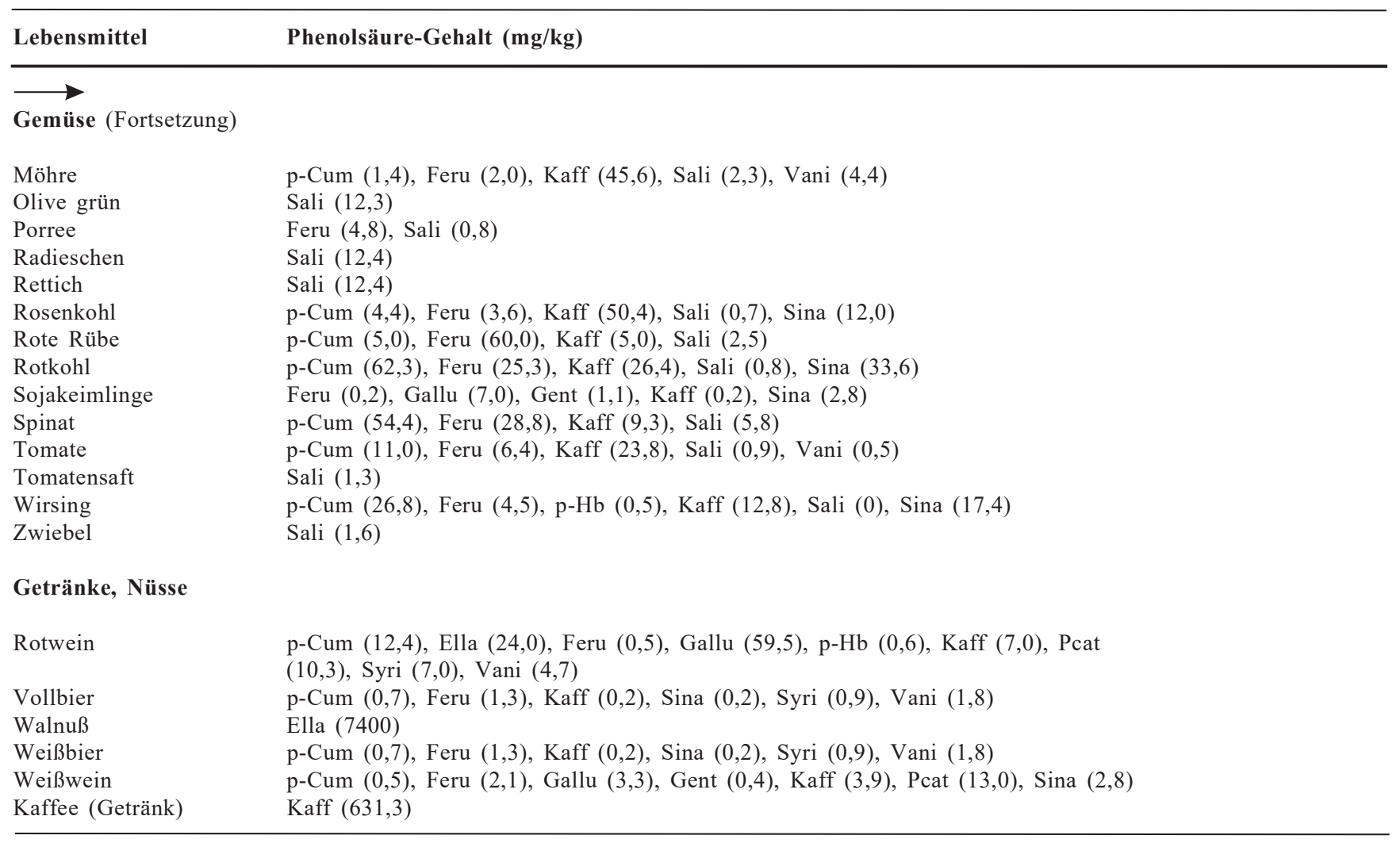

$(p-C u m=p$-Cumarsäure, Ella $=$ Ellagsäure, Feru $=$ Ferulasäure, Gallu $=$ Gallussäure, Gent $=$ Gentisinsäure, p-Hb $=$ p-Hydroxybenzoesäure, Kaff $=$ Kaffeesäure, Pcat $=$ Protocatechusäure, Sali $=$ Salicylsäure, Sina $=$ Sinapinsäure, Syri $=$ Syringasäure, Vani $=$ Vanillinsäure $)$

\section{Literaturquellen:}

Amiot MJ et al. (1995) J Agric Food Chem 43:1132-1137; Boyle JA et al. (1990) Am J Enol Vitic 41:43-47; Brenes M et al. (1992) J Food Sci 58:347-350; Daniel EM et al. J Food Composition Analysis 2:338-349; Delage E (1991) J Chromatogr 555:125-136; Frankel EN et al. (1995) J Agric Food Chem 43:890-894; Gao I et al. (1995) J Agric Food Chem 43:343-346; Gil MI et al. (1995) Z Lebensm Unters Forsch 200:278-281; Gorsel van H et al. (1992) J Agric Food Chem 40:784-789; Häberle M (1987) Ernährungs-Umschau 34:287-296; Hayes PJ (1987) Analyst 112:1205-1207; Herrmann K (1989) Crit Rev Food Sci Nutrit 28:315-347; Herrmann K (1989) Erwerbsobstbau 31:185-189; Herrmann K (1990) Ernährungs-Umschau 37:108-112; Herrmann K (1992) Flüssiges Obst 59:66-70; Hollman PCH \& Venema DP (1993) The content of the potentially anticarcinogenic ellagic acid in plant foods. In: Food and cancer prevention: chemical and biological aspects. Waldron KW, Johnson IT, Fenwick GR (eds) Royal Society of Chemistry, Cambridge, pp 203-208; Kermasha S et al. (1995) Food Res Int 28:245-252; Laszlavik M et al. (1995) Am J Enol Vitic 46:67-74; Lee HS \& Wrolstad RE (1988) J Assoc Off Anal Chem 71:789-794; Lin TY \& Vine RP (1990) J Food Sci 55:1607-1613; Macheix JJ et al. (1990) Fruit Phenolics. CRC Press, Boca Raton, pp 19-24; Maier HG \& Grimsehl A (1982) Kaffee Tee Markt 32:3-6; Mayén M et al. (1995) Am J Enol Vitic 46:255-261; McRae KB \& Lidster PD (1990) J Sci Food Agric 50:329-342; Ong BY \& Nagel CW (1978) J Chromatogr 157:345-355; Oszmianski J \& Lee CY (1990) Am J Enol Vitic 41:204-206; Pérez-Ilzarbe J et al. (1991) Z Lebensm Unters Forsch 192:551-554; Ping L et al. (1993) Biomed Environ Sci 6:389-398; Risch B \& Herrmann K (1988) Z Lebensm Unters Forsch 186:225-230; Rommel A \& Wrolstad RE (1993) J Agric Food Chem 41:1951-1960; Scherz H \& Souci SW (1994) Die Zusammensetzung der Lebensmittel, Nährwert-Tabellen. CRC Press, Boca Raton; Senter SD \& Callahan A (1990) J Food Sci 55:1585-1602; Simón de BF et al. (1992) J Agric Food Chem 40:1531-1535; Spanos GA \& Wrolstad RE (1990) J Agric Food Chem 38:817-824; Spanos GA \& Wrolstad RE (1992) J Agric Food Chem 40:1478-1487; Stöhr H \& Herrmann K (1975) Z Lebensm Unters Forsch 159:341-348; Swain AR et al. (1985) J Amer Diet Assoc 85:950-960.

hierfür die repräsentativ ausgewählten Personen des Erhebungsgebietes Ober-/Mittel-/Unterfranken (Erhebungsreihe 16) herangezogen (Quelle: Public Use File: NVS und VERA). Für die Altersgruppe ,19-24 Jahre“ wurden 52 Personen (27 Frauen, 25 Männer) in die Untersuchung aufgenommen, für die Altersgruppe ,25-49 Jahre“ waren es 67 Personen (36 Frauen, 31 Männer). Insgesamt beinhaltet das Studienkollektiv 119 Personen, davon 63 Frauen und 56 Männer. Für weitere Angaben zum Kollektiv wird auf eine frühere Veröffentlichung zur Flavonoidzufuhr verwiesen (12). 


\section{Auswertung}

Für die Auswertung der Ernährungsprotokolle wurden die Werte der Phenolsäure-Datenbank in die Datengrundlage der Software Prodi III plus (Wissenschaftliche Verlagsgesellschaft, Stuttgart) übertragen. Für einige wenige Lebensmittel (Konfitüre, Trockenfrüchte, Tomatenketchup) wurden Werte mit Hilfe der Analysendaten der Rohprodukte errechnet. Die Zufuhr von Phenolsäuren im Kollektiv ist anhand verschiedener Parameter der deskriptiven Statistik dargestellt. Die Überprüfung des Einflusses von Alter (Altersgruppe „19-24 Jahre“ bzw. „25-49 Jahre") $^{\text {") }}$ und Geschlecht auf die Zufuhr erfolgte anhand des verteilungsfreien Mann-Whitney-Tests mit einer Irrtumswahrscheinlichkeit von $\mathrm{p}<0,05$.

\section{Ergebnisse}

Die mittlere Zufuhr von Phenolsäuren im Gesamtkollektiv beträgt $222 \mathrm{mg}$ pro Tag mit einer sehr großen Schwankungsbreite von $8,7 \mathrm{mg}$ bis $989,0 \mathrm{mg}$ pro Tag (nicht dargestellt). An der mittleren Zufuhr der Hydroxybenzoesäuren $(11,3 \mathrm{mg} / \mathrm{d})$ sind die hier erfaßten Phenolsäuren gleichmäßig beteiligt, im Gegensatz dazu dominiert bei der Zufuhr der Hydroxyzimtsäuren $(211,0 \mathrm{mg} / \mathrm{d}) \mathrm{klar}$ die Kaffeesäure. Auch bezogen auf die Summe aller Phenolsäuren im Gesamtkollektiv macht die Kaffeesäure mit 205,5 mg/d den Hauptanteil aus.

Die getrennte Auswertung für Männer und Frauen (Tab. 2) zeigt signifikante Geschlechts-Unterschiede für die mittlere Zufuhr von Vanillin-, Chlorogen-, Kaffee-, Ferula- und Sinapinsäure bzw. der Summe der Hydroxyzimtsäuren und der Summe aller Phenolsäuren. Die Kaffeesäure und Chlorogensäure-Zufuhr ist bei den Frauen deutlich höher. Als Folge sind die mittleren Summenwerte der Phenolsäuren und der Hydroxyzimtsäuren bei den Frauen mit 244,8 bzw. 234,0 mg/d größer als bei den Männern mit 196,8 bzw. 185,1 mg/d. Für alle anderen erfaßten Phenolsäuren liegt die mittlere Zufuhr der Männer signifikant bzw. tendenziell höher als die der Frauen. Für die Kaffeesäure als Vertreter der Hydroxyzimtsäuren beträgt bereits das Minimum 4,8 mg/d (Männer) bzw. $6,2 \mathrm{mg} / \mathrm{d}$ (Frauen). Die Mittelwerte der Zufuhr liegen bei allen Phenolsäuren höher als die Mediane, dies gilt für die Männer ebenso wie für die Frauen.

Vergleicht man die Ergebnisse in den beiden Altersgruppen ,19-24 Jahre“ und 25-49 Jahre“, so findet man für die Zufuhr von Kaffeesäure und Chlorogensäure und in der Folge für die Summe der Hydroxyzimtsäuren und die Summe der Phenolsäuren signifikante Altersunterschiede. Hierbei liegt der Mittelwert in der Altersgruppe „25-49 Jahre“ (Summe der Phenolsäuren 259,1 mg/d) höher als der in der Altersgruppe ,25-24 Jahre“ (Summe der Phenolsäuren 174,6 mg/d) (nicht dargestellt).

Die prozentualen Anteile von Lebensmitteln und Lebensmittelgruppen an der Zufuhr der einzelnen Phenolsäuren gehen aus Tabelle 3 hervor. Als Quelle für die Kaffeesäure dominiert Kaffee mit einem durchschnittlichen Beitrag von $92 \%$. Gallussäure wird nahezu ausschließlich mit Rot- und Weißwein (93\%) aufgenommen, ähnlich dominierend ist für die p-HydroxybenzoesäureZufuhr die Lebensmittelgruppe Obst/-produkte/-säfte (93

Tab. 2 Phenolsäure-Zufuhr $(\mathrm{mg} / \mathrm{d})$ von Männern $(\mathrm{n}=56)$ und Frauen $(\mathrm{n}=63)$ eines bayerischen Teilkollektivs der NVS

\begin{tabular}{|c|c|c|c|c|c|c|c|c|c|c|}
\hline \multirow[b]{2}{*}{ Phenolsäure } & \multicolumn{5}{|c|}{ Männer } & \multicolumn{5}{|c|}{ Frauen } \\
\hline & $\begin{array}{c}\text { Median } \\
(\mathrm{mg} / \mathrm{d})\end{array}$ & $\begin{array}{l}\text { Min. } \\
(\mathrm{mg} / \mathrm{d})\end{array}$ & $\begin{array}{c}10 \%-90 \% \\
(\mathrm{mg} / \mathrm{d})\end{array}$ & $\begin{array}{l}\text { Max. } \\
(\mathrm{mg} / \mathrm{d})\end{array}$ & $\underset{(\mathrm{mg} / \mathrm{d})}{\operatorname{Mean} \pm \text { SEM }}$ & $\begin{array}{l}\text { Median } \\
(\mathrm{mg} / \mathrm{d})\end{array}$ & $\begin{array}{l}\text { Min. } \\
(\mathrm{mg} / \mathrm{d})\end{array}$ & $\begin{array}{c}10 \%-90 \% \\
(\mathrm{mg} / \mathrm{d})\end{array}$ & $\begin{array}{l}\text { Max. } \\
(\mathrm{mg} / \mathrm{d})\end{array}$ & $\underset{(\mathrm{mg} / \mathrm{d})}{\operatorname{Mean}} \pm \underset{(\mathrm{mg} / \mathrm{d})}{\mathrm{SEM}}$ \\
\hline p-Hydroxybenzoesäure & 0,17 & 0,00 & $0,02-0,81$ & 1,69 & $0,29 \pm 0,05$ & 0,13 & 0,00 & $0,04-0,55$ & 2,96 & $0,24 \pm 0,05$ \\
\hline Protocatechusäure & 0,27 & 0,00 & $0,01-2,33$ & 4,17 & $0,63 \pm 0,13$ & 0,25 & 0,00 & $0,02-1,46$ & 3,44 & $0,53 \pm 0,09$ \\
\hline Vanillinsäure & 0,70 & 0,03 & $0,11-2,51$ & 4,09 & $1,06 \pm 0,13 *$ & 0,31 & 0,24 & $0,05-1,12$ & 2,03 & $0,47 \pm 0,06^{*}$ \\
\hline Salicylsäure & 1,41 & 0,11 & $0,45-3,18$ & 4,40 & $1,62 \pm 0,14$ & 1,34 & 0,21 & $0,37-3,61$ & 10,27 & $1,87 \pm 0,23$ \\
\hline Gentisinsäure & 0,19 & 0,00 & $0,00-0,68$ & 2,20 & $0,26 \pm 0,04$ & 0,16 & 0,00 & $0,02-0,54$ & 1,10 & $0,22 \pm 0,03$ \\
\hline Gallussäure & 0,20 & 0,00 & $0,00-4,88$ & 17,83 & $1,57 \pm 0,47$ & 0,14 & 0,00 & $0,00-3,94$ & 5,74 & $0,92 \pm 0,19$ \\
\hline Ellagsäure & 1,27 & 0,00 & $0,00-10,24$ & 81,45 & $4,95 \pm 1,57$ & 0,82 & 0,00 & $0,00-12,35$ & 91,80 & $5,43 \pm 2,01$ \\
\hline Syringasäure & 1,33 & 0,00 & $0,23-2,76$ & 4,48 & $1,40 \pm 0,13$ & 1,04 & 0,00 & $0,02-2,57$ & 3,47 & $1,12 \pm 0,11$ \\
\hline $\begin{array}{l}\text { Summe Hydroxy- } \\
\text { benzoesäuren }\end{array}$ & 6,98 & 1,11 & $2,70-25,71$ & 90,78 & $11,78 \pm 1,85$ & 6,19 & 0,64 & $2,14-20,10$ & 94,15 & $10,79 \pm 2,06$ \\
\hline Kaffeesäure & 113,78 & 4,78 & $15,72-381,09$ & 983,18 & $179,00 \pm 25,5^{*}$ & 209,77 & 6,19 & $40,03-439,17$ & 787,06 & $229,05 \pm 19,6 *$ \\
\hline Chlorogensäure ${ }^{\#}$ & 48,61 & 1,85 & $15,12-168,65$ & 383,97 & $77,66 \pm 9,91 *$ & 93,33 & 4,59 & $24,54-178,96$ & 319,27 & $100,54 \pm 7,93 *$ \\
\hline p-Cumarsäure & 3,14 & 0,60 & $1,56-5,79$ & 26,66 & $3,78 \pm 0,47$ & 2,74 & 0,41 & $0,83-6,08$ & 13,17 & $3,11 \pm 0,30$ \\
\hline Ferulasäure & 1,84 & 0,25 & $0,80-3,03$ & 5,12 & $1,90 \pm 0,12 *$ & 1,25 & 0,11 & $0,46-\quad 2,08$ & 15,33 & $1,53 \pm 0,24 *$ \\
\hline Sinapinsäure & 0,30 & 0,00 & $0,04-0,81$ & 2,34 & $0,39 \pm 0,05^{*}$ & 0,15 & 0,00 & $0,02-\quad 0,85$ & 1,73 & $0,28 \pm 0,04 *$ \\
\hline $\begin{array}{l}\text { Summe Hydroxy- } \\
\text { zimtsäuren }\end{array}$ & 120,66 & 6,23 & $22,28-390,17$ & 987,10 & $185,07 \pm 25,5^{*}$ & 211,69 & 9,94 & $42,91-442,85$ & 791,99 & $233,98 \pm 19,6^{*}$ \\
\hline Summe Phenolsäuren & 128,62 & 8,67 & $29,97-436,66$ & 988,96 & $196,84 \pm 25,8^{*}$ & 214,21 & 13,34 & $47,24-464,21$ & 796,24 & $244,77 \pm 20,0^{*}$ \\
\hline
\end{tabular}

* signifikant unterschiedliche Mittelwerte für Männer und Frauen, p < 0,05, Mann-Whitney-Test

* Chlorogensäure (5-Caffeoylchinasäure) als bekanntester Vertreter der Kaffeesäuren extra ausgewiesen 
Tab. 3 Durchschnittliche Zufuhr (mg/d) von Hydroxybenzoesäuren (HBS) und Hydroxyzimtsäuren (HZS) und durchschnittliche prozentuale Anteile ausgewählter Lebensmittel/-gruppen an der Gesamtzufuhr von Hydroxybenzoesäuren und Hydroxyzimtsäuren in einem bayerischen Teilkollektiv der NVS (56 Männer und 63 Frauen)

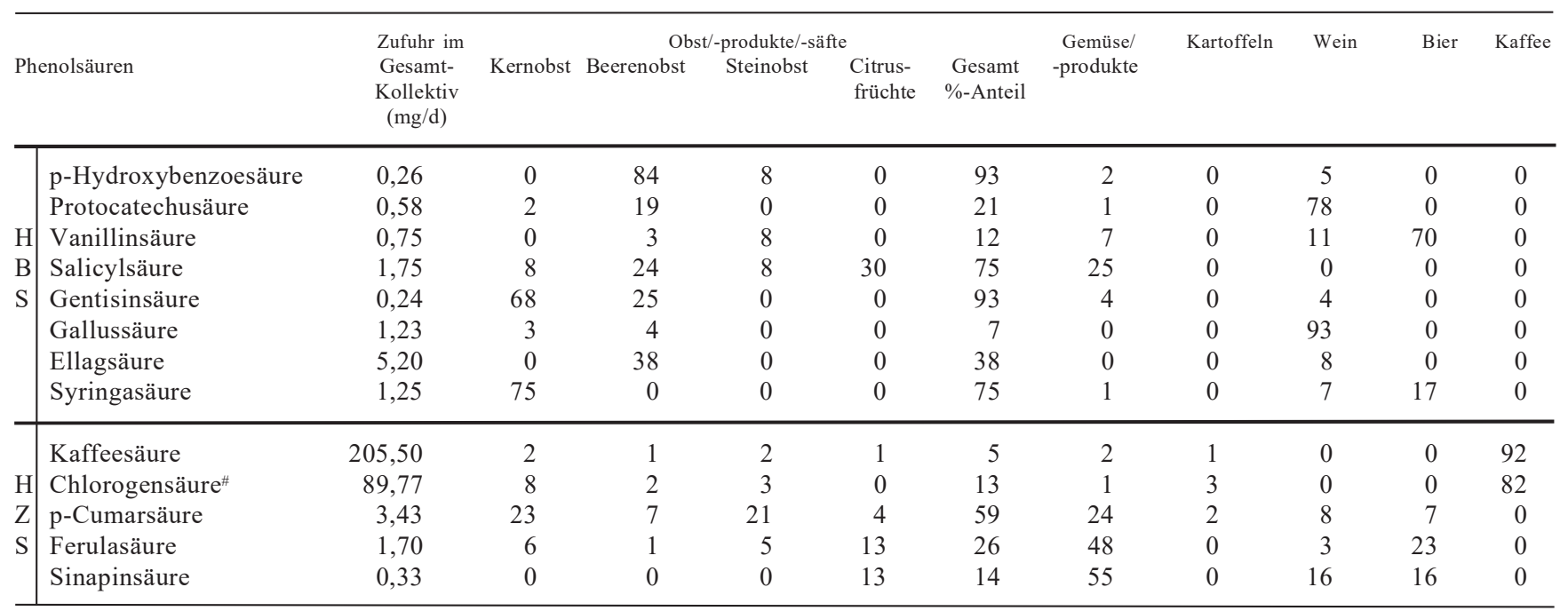

* Chlorogensäure (5-Caffeoylchinasäure) als bekanntester Vertreter der Kaffeesäuren extra ausgewiesen

\%) oder für Ellagsäure Beerenobst (38\%) und Walnüsse (54 \%). Neben dem Getränk Kaffee ist die Gruppe Obst/produkte/-säfte insgesamt der wichtigste Lieferant für Hydroxybenzoe- und Hydroxyzimtsäuren.

Die signifikanten Geschlechts- bzw. Altersunterschiede für Kaffeesäure, Summe Hydroxyzimtsäuren und Summe der Phenolsäuren werden ausschließlich durch den höheren Kaffeekonsum der Frauen bzw. der Personen in der Altersgruppe ,25-49 Jahre“ verursacht (Männer 19-24 Jahre: $208 \mathrm{ml} / \mathrm{d}, 25-49$ Jahre: $303 \mathrm{ml} / \mathrm{d}$; Frauen 19-24 Jahre: $255 \mathrm{ml} / \mathrm{d}, 25-49$ Jahre: $392 \mathrm{ml} / \mathrm{d}$ ). Berücksichtigt man den Anteil des Kaffees an der PhenolsäureZufuhr nicht, so entfallen diese Alters- und Geschlechtsunterschiede.

\section{Diskussion}

Um die Qualität der verwendeten Datenbank zu beurteilen, wurde die mittlere Energieaufnahme über die Gruppe „Obst/-produkte/-säfte“ bzw. „Gemüse/-produkte/-säfte“ (incl. Kartoffeln) mit der Energiemenge verglichen, die ausschließlich über Lebensmittel mit Phenolsäure-Analysendaten dieser beiden Gruppen zugeführt wurden. Über Lebensmittel mit Analysendaten werden hierbei $85 \%$ der Energiezufuhr der Gruppe „Obst/-produkte/-säfte“ gedeckt. Für die Gruppe „Gemüse/-produkte/-säfte“ (incl. Kartoffeln) beträgt dieser Wert $89 \%$. Bei einer Zufuhr von $110 \mathrm{kcal} / \mathrm{d}$ aus der Gruppe „Obst/-produkte/-säfte“ und $87 \mathrm{kcal} / \mathrm{d}$ aus der Gruppe „Gemüse/-produkte/-säfte“ werden insgesamt $87 \%$ der Energie dieser beiden Gruppen über Lebensmittel aufgenommen, für die Analysendaten vorliegen. Die verbleibenden $13 \%$ entfallen auf viele verschiedene Lebensmittel, die jeweils in nur geringen Mengen verzehrt werden. Es lassen sich also keine bestimmten Lebensmittel pflanzlichen Ursprungs nennen, deren Analyse die Lücke weitgehend schließen würde.

Eine weitere Quelle für die Phenolsäurezufuhr sind Getreideprodukte. In der Aleuronschicht, Schale und Keimling des Weizenkorns findet man Ferulasäure in hohen Konzentrationen, im stärkehaltigen Endosperm dagegen nur in Spuren $(15,16)$. Exakte Angaben über den Ferulasäuregehalt von verschiedenen Weizenmehltypen, die üblicherweise in der menschlichen Ernährung verwendet werden, sind aber nicht vorhanden. Ebenso fehlen Angaben für weitere Getreidesorten. Es ist folglich davon auszugehen, daß die Zufuhr von Ferulasäure durch den Verzehr von Getreideprodukten, insbesondere Vollkornprodukten, höher liegt als hier ausgewiesen (Tab. 2).

Da in Lebensmitteln pflanzlichen Ursprungs Sorte, Jahreszeit, Anbau und Reifezustand einen großen Einfluß auf den Phenolsäuregehalt haben, könnte die berechnete Zufuhr von der tatsächlichen Zufuhr deutlich abweichen. Selbst die Größe von Früchten (z.B. Äpfeln) beeinflußt den Phenolsäuregehalt, da dieser im Schalenbereich gröBer ist und so kleine Früchte höhere Gehalte aufweisen als große (3). Zudem handelt es sich bei den hier verwendeten Analysendaten um die Werte von rohen Produkten und es ist wenig über die Zubereitungsverluste von Phenolsäuren bekannt. Mit den natürlichen Schwankungen der Gehalte in der Pflanze und den küchentech- 
nischen Verlusten bestehen also zwei schwer abschätzbare Einflußfaktoren auf die Zufuhr der Hydroxyzimtsäuren und Hydroxybenzoesäuren. Die Beurteilung der Zufuhrdaten sollte daher auf Gruppenebene und nicht auf Personenebene erfolgen.

In der vorliegenden Datenbank werden nur die „Grundkörper" der einzelnen Vertreter der Phenolsäuren dargestellt, obwohl sie in dieser Form in der Pflanze bzw. im Lebensmittel normalerweise nicht vorliegen. Bei einem Teil der Untersuchungen, welche die Grundlage für diese Datenbank darstellen, wird nicht nach den tatsächlich vorliegenden Estern und Glycosiden der Phenolsäuren bzw. den verschiedenen Isomeren differenziert, sondern es werden die Phenolsäuren, wie sie nach Säurehydrolyse vorliegen, dargestellt. Wollte man also alle tatsächlich vorliegenden Derivate einer Phenolsäure darstellen, würden diese Analysen-Quellen entfallen. Ein solches Vorgehen erscheint auch nicht praktikabel, da durch die Kombination der verschiedenen Säure- bzw. Glycosidreste und durch die möglichen Isomere die Zahl der Verbindungen sehr unübersichtlich wird und zum momentanen Zeitpunkt zu wenig Informationen über Unterschiede in der biologischen Wirkung vorliegen.

Die unterschiedlich hohe Zufuhr an Kaffeesäure bei Männern und Frauen (siehe Tab. 2) und auch in beiden Altersgruppen läßt sich durch den höheren Kaffeekonsum der Frauen bzw. der Personen in der Altersgruppe ,19-49 Jahre" erklären. Bezüglich der Höhe des Kaffeekonsums stimmen die vorliegenden Ergebnisse mit den in der Nationalen Verzehrsstudie ausgewiesenen Werten gut überein, der Geschlechterunterschied ist dort jedoch geringer (1). Berücksichtigt man nur die Zufuhr über alle anderen Lebensmittel, aber ohne den Kaffee, so entfallen die hier genannten Geschlechts- und Altersunterschiede für Kaffeesäure, Summe der Hydroxyzimtsäuren und Gesamtsumme der Phenolsäuren. Die dominierende Bedeutung des Kaffees für die Phenolsäurezufuhr läßt sich mit der Bedeutung des Tees für die Flavonoid- (bzw. Flavonol-) Zufuhr in niederländischen und japanischen Populationen vergleichen (5).
Für die errechnete mittlere Zufuhr von 5,2 mg/d Ellagsäure existiert für die Niederlande mit $6 \mathrm{mg} / \mathrm{d}$ ein vergleichbarer Wert (6). In beiden Populationen ist die Ellagsäure-Zufuhr durch die hohen Ellagsäure-Gehalte von Beerenobst und Walnüssen bedingt.

Mögliche präventive Wirkungen der Phenolsäuren in der menschlichen Ernährung ergeben sich aus ihrer antikanzerogenen und antioxidativen Wirkung. Durch den Schutz von LDL-Partikeln vor oxidativer Modifikation könnten sie präventiv gegen die Arterioskleroseentstehung wirken und durch ihre antikanzerogene Wirkung, z.B. Hemmung der Nitrosaminbildung, die Krebsentstehung hemmen. Eine verminderte Bildung von Hydroxylradikalen in vitro konnte für Kaffee-, Chlorogen- und Gallussäure gezeigt werden (8). Kaffee-, Chlorogen-, Ellag- und Protocatechusäure (11) zeigen ebenso wie Kaffee-, Ferula- und p-Cumarsäure (13) in vitro einen Schutzeffekt gegenüber der oxidativen Veränderung von humanen LDL-Partikeln. Hydroxyzimt- und Hydroxybenzoesäuren stellen in vitro (14) ebenso wie Kaffee- und Ferulasäure im Tierversuch (10) potente Inhibitoren der Nitrosaminbildung dar. Im Tierversuch konnte für Chlorogen-, Kaffee-, Ferula- und Protocatechusäure eine antikanzerogene Wirkung gezeigt werden $(7,19,20)$. Epidemiologische Untersuchungen, die den Zusammenhang zwischen der Phenolsäure-Zufuhr und der Entstehung koronarer Herzerkrankungen (KHK) oder der Entstehung von Krebs beim Menschen untersuchen, wie sie für die Flavonoid-Zufuhr bereits vorliegen $(5,9)$, gibt es bisher nicht. Es existieren jedoch bereits einzelne Studien, die den Zusammenhang von Kaffeekonsum und KHK bzw. Krebs untersuchen. Dabei wurde weder für die Entstehung von KHK noch von Krebs ein eindeutiger Einfluß des Kaffeekonsums gefunden $(17,18,21,22)$. Die Wirkungen von Kaffeekonsum und Kaffeesäure-Zufuhr lassen sich in epidemiologischen Studien nur schwer trennen. Möglicherweise könnte in Gruppen mit geringem Kaffeekonsum - und damit geringer Kaffeesäure-Zufuhr über Kaffee - die Wirkung von Phenolsäuren deutlicher hervortreten.

\section{Literatur}

1. Adolf T, Schneider R, Eberhardt W, Hartmann S, Herwig A, Heseker $\mathrm{H}$, Hünchen K, Kübler W, Matiaske B, Moch KJ, Rosenbauer J (1995) Ergebnisse der Nationalen Verzehrsstudie (1985- 1988) über die Lebensmittelund Nährstoffaufnahme in der Bundesrepublik Deutschland. Band XI der VERA-Schriftenreihe. Wissenschaftlicher Fachverlag Dr. Fleck, Niederkleen

2. Deutsche Gesellschaft für Ernährung (Hrsg) (1996) Ernährungsbericht 1996. Druckerei Henrich GmbH, Frankfurt/M, p 226
3. Herrmann K (1989) Vorkommen und Gehalte der Phenolcarbonsäuren in Obst. Erwerbsobstbau 31:185-189

4. Herrmann K (1992) Über die Gehalte der hauptsächlichen Pflanzenphenole im Obst. Flüssiges Obst 59:66-70

5. Hertog MGL, Kromhout D, Aravanis C, Blackburn H, Buzina R, Fidanza F, Giampaoli S, Jansen A, Menotti A, Nedeljocovic S, Pekkarinen M, Simic BS, Toshima H, Fesken EJM, Hollman PCH, Katan MB (1995) Flavonoid intake and longterm risk of coronary heart disease and cancer in the Seven Countries
Study. Arch Intern Med 155:381386

6. Hollman PCH, Venema DP (1992) The content of the potentially anticarcinogenic ellagic acid in plant foods (Wageningen, Netherlands). Poster at the International Conference on "Food and cancer prevention", Norwich, UK

7. Huang MT, Smart RC, Wong CQ, Conney AH (1988) Inhibitory effect of curcumin, chlorogenic acid, caffeic acid, and ferulic acid on tumor promotion in mouse skin by 12-O-tetradecanoylphorbol-13-acetate. Cancer Res 48:5941-5946 
8. Iwahashi H, Ishii T, Sugata R, Kido R (1990) The effects of caffeic acid and its related catechols on hydroxyl radical formation by 3-hydroxyanthranilic acid, ferric chloride, and hydrogen peroxide. Arch Biochem Biophys 276: 242-247

9. Katan MB (1997) Flavonoids and heart disease. Am J Clin Nutr 65:1542-1543

10. Kuenzig W, Chau J, Norkus E, Holowaschenko $\mathrm{H}$, Newmark $\mathrm{H}$, Mergens W, Conney AH (1994) Caffeic and ferulic acid as blockers of nitrosamine formation. Carcinogenesis 5:309-313

11. Laranjinha JA, Almeida LM, Madeira VM (1994) Reactivity of dietary phenolic acids with peroxyl radicals: antioxidant activity upon low density lipoprotein peroxidation. Biochem Pharmacol 48:487-494

12. Linseisen J, Radtke J, Wolfram G (1997) Flavonoidzufuhr Erwachsener in einem bayerischen Teilkollektiv der Nationalen Verzehrsstudie. Z Ernährungswiss 36:403-412

13. Nardini M, D Aquino M, Tomassi G, Gentili V, Di-Felice M, Scaccini C (1995) Inhibition of human low- density lipoprotein oxidation by caffeic acid and other hydroxycinnamic acid derivatives. Free Radic Biol Med 19:541-552

14. Ping L, Huai-Zhou W, Xu-Qing W, Yong-Ning W (1994) The blocking effect of phenolic acid on N-nitrosomorpholine formation in vitro. Biomed Environ Sci 7:68-78

15. Pussayanawin V, Wetzel DL (1987) High-performance liquid chromatographic determination of ferulic acid in wheat milling fractions as a measure of bran contamination. J Chromatogr $391: 243-255$

16. Pussayanawin V, Wetzel DL, Fulcher RG (1988) Fluorescence detection and measurement of ferulic acid in wheat milling fractions by microscopy and HPLC. J Agric Food Chem 36: $515-520$

17. Rosenberg L, Miller DR, Helmrich SP, Kaufman DW, Schottenfeld D, Stolley PD, Shapiro S (1985) Breast cancer and the consumption of coffee. Am J Epidemiol 122:391-399

18. Rosenberg L, Werler MM, Palmer JR, Kaufman DW, Warshauer ME, Stolley
PD, Shapiro S (1989) The risk of cancer of the colon and rectum in relation of coffee consumption. Am J Epidemiol 130:895-903

19. Tanaka $\mathrm{T}$, Kojima $\mathrm{T}$, Kawamori $\mathrm{T}$, Mori H (1995) Chemoprevention of digestive organs carcinogenesis by natural product protocatechuic acid. Cancer 75:1433-1439

20. Tanaka T, Kojima $\mathrm{T}$, Kawamori $\mathrm{T}$, Yoshimi N, Mori H (1993) Chemoprevention of diethylnitrosamine-induced hepatocarcinogenesis by a simple phenolic acid protocatechuic acid in rats Cancer Res 53:2775-2779

21. Thelle DS, Heyden S, Fodor JG (1987) Coffee and cholesterol in epidemiological and experimental studies. Atherosclerosis 67:97-103

22. Willett WC, Stampfer MJ, Manson JE, Colditz GA, Rosner BA, Speizer FE, Hennekens CH (1996) Coffee consumption and coronary heart disease in women. A ten-year follow-up. JAMA 275:458-462 